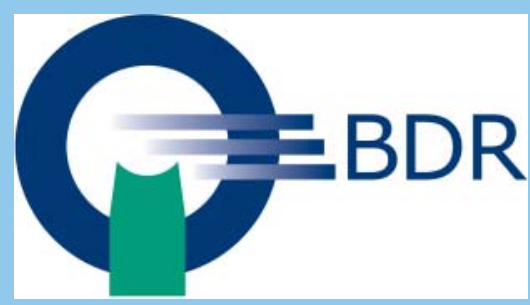

\title{
Interventionelle Radiologie in Salzburg - Bericht vom IROS 2018
}

Der IROS präsentierte sich auch 2018 als erster überregionaler radiologischer Kongress des Jahres, Salzburg, 11.-13.Januar 2018, nicht nur den ausgewiesenen Spezialisten der interventionellen Radiologie, sondern auch der großen Anzahl an Kolleginnen und Kollegen, die unter anderem interventionelle Radiologie in ihrem Alltag durchführen. Mit seinem breiten Spektrum der gesamten interventionellen Radiologie, die neben der Gefäßmedizin vor allem auch die Tumortherapie und die vaskuläre Notfallversorgung umfasst, wird er daher von den Radiologen Deutschlands, Österreichs und der Schweiz, deren Dreiländertagung er ist, weiterhin sehr gut angenommen. Trotz der wenige Wochen später stattfindenden, allerdings ganz auf vaskuläre Fragen abgestellten „Parallelveranstaltung“ des LINC in Leipzig. Geschätzt wird dabei vor allem auch die überschaubarere, ja fast familiäre Atmosphäre des Kongresses, insbesondere wenn er, wie in diesem Jahr, in Salzburg stattfindet. Diese Atmosphäre erlaubt einen sehr engen Austausch der Teilnehmer untereinander, in den Sitzungen ebenso wie in den Pausen. Ein großes Plus stellt die unmittelbar vor den Konferenzräumen stattfindende Industrieausstellung dar, so dass zwischen den Sitzungen genug Möglichkeit ist die Stände zu besuchen und Weiter- bzw. Neuentwicklungen zu diskutieren.

Im Programm wurden die im Vorjahr begonnenen vorsichtigen Modifizierungen weiter vorangetrieben. So fanden in diesem Jahr erstmals keine echten Live-Case-Präsentationen mehr statt. Sie wurden ersetzt durch Videoaufzeichnungen entsprechender Eingriffe. Diese waren im Vorfeld aufgezeichnet und auch geschnitten worden, wobei die jeweiligen Operateure als Moderatoren der Sitzung anwesend und damit für das Publikum für Fragen zur Verfügung standen. Die Voraufzeichnung verringert deutlich die Anspannung beim die Intervention durchführenden Team und ist der Gesamtdiskussion sicherlich förderlich. Für Fragen aus dem Publikum stand durchgehend die wieder deutlich verbesserte Kongress-App zur Verfügung. Damit können wiederkehrende und damit häufige Fragen vom Moderator zusammengefasst diskutiert und der Fluss der Präsentation und Diskussion gestrafft werden.

Besonders ausgebaut wurden in diesem Jahr auch die Basiskurse für weniger erfahrene Kolleginnen und Kollegen im Rahmen des Zertifizierungsprogramms der DeGIR, aber auch darüber hinaus. Dies führt zwar zu einer weiteren Zersplitterung des Kongresses durch eine Zunahme der Parallelveranstaltungen, macht den Kongress andererseits aber auch für die „NochNicht-Spezialisten“ interessant. In diesem Zusammenhang ist der Ausbau des MTRAProgramms besonders zu erwähnen. Für das Assistenzpersonal steht jetzt an einem der Kongresstage ein eigenes Programm zur Verfügung, was sicherlich zur Attraktivität des Kongresses in dieser Berufsgruppe beitragen wird.

Auch die Posterausstellung wurde wieder sehr gut angenommen und in diesem Jahr durch eine sogenannte „Poster-Safari“ ergänzt, in der die Autoren ihre Ergebnisse in einem moderierten, virtuellen Posterausstellungsbesuch kurz präsentieren konnten. Auch neu im Programm die „Breaking News“, die ganz aktuelle Ergebnisse zum Zusammenhang von Thermoablation und Immunstimulation, zur Mikrowellenablation von Schilddrüsenknoten und zur Lithoplastie bei der pAVK berichteten.

Kaum ein Verfahren der interventionellen Tumortherapie wird derzeit so kontrovers diskutiert wie die SIRT in der Behandlung von Lebermetastasen. Aktuelle Studien zeigen zwar ein statistisch signifikantes Ansprechen auf die Behandlung, allerdings im Gesamt-Outcome, nach derzeitiger Datenlage, keinen Vorteil gegenüber den systemischen Therapieformen. Zu berücksichtigen sind dabei jedoch die Auswahl der Patientenkollektive, die generell erst sehr spät im Krankheitsverlauf eingesetzte Therapie der SIRT und die Zusammensetzung der Patientengruppen. Mit Interesse wird darüber hinaus die künftig mögliche SIRT mit Holmium-166 statt Itrium-90 verfolgt, zu der erste Studienergebnisse präsentiert wurden. Es bleibt abzuwarten, ob die nuklidimanenten Vorteile des Holmiums (Sichtbarkeit in der T2w der MRT/Darstellbarkeit im SPECT durch einen signifikanten Gamma-Strahlungsanteil) das Verfahren insgesamt befeuern können und die Therapieergebnisse vergleichbar zur bisherigen Itrium-Therapie sind.

Einen Publikumsmagnet stellen die seit einigen Jahren angebotenen Formate „Ein Fall, der mich nicht schlafen ließ“ jeweils als Abschluss der ersten beiden Kongresstage und die zum Finale der Veranstaltung am Samstag-Mittag präsentierte „M\&M-Konferenz“ dar. Dabei berichten ausgewiesene Experten über Erfahrungen mit interventionellen Eingriffen, die zu einer besonderen Herausforderung aufgrund der Komplexität des Falles oder eingetretener Komplikationen wurden. Es ist dabei den Referenten besonders hoch anzurechnen neben schicksalshaften Verläufen auch eigene Fehleinschätzungen öffentlich zu diskutieren. Sicherlich jedoch Formate, die erheblich zum Erfahrungsgewinn der Teilnehmer der Sitzungen beitragen und deshalb auch stets besonderen Zuspruch erfahren. 
Zum Ende des, wie immer vom erfahrenen Team des ECR perfekt organisierten Kongresses konnten die Vorsitzenden des Programm-Komitees Prof. Pereira (Heilbronn) und Prof. Schoder (Wien) somit auch ein sehr positives Fazit ziehen. Der Kongress entwickelt sich stetig fort, ist ein Anziehungspunkt für die Radiologen aus den drei veranstaltenden Fachgesellschaften vom Assistenzarzt mit wenig Erfahrung bis zum langjährig versierten Interventionalisten, ebenso wie für das Assistenzpersonal der MTRA, MFA und Angioschwestern. Er hat damit in jedem Fall seine Berechtigung gegenüber dem wenig später stattfindenden LINC und stellt das Pendant im Frühjahr zur CIRSE im Herbst dar.

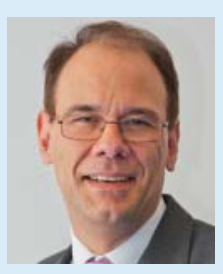

Prof. Hermann Helmberger, München 